\title{
TD Kernel DM+V: Time-Dependent Statistical Gas Distribution Modelling on Simulated Measurements
}

Sahar Asadi, Sepideh Pashami, Amy Loutfi, Achim J. Lilienthal

AASS Research Center, Örebro University, Örebro, Sweden

\{first name\}.\{surname\}@oru.se

\section{Summary}

To study gas dispersion, several statistical gas distribution modelling approaches have been proposed recently. A crucial assumption in these approaches is that gas distribution models are learned from measurements that are generated by a time-invariant random process. While a time-independent random process can capture certain fluctuations in the gas distribution, more accurate models can be obtained by modelling changes in the random process over time. In this work we propose a time-scale parameter that relates the age of measurements to their validity for building the gas distribution model in a recency function. The parameters of the recency function define a time-scale and can be learned. The time-scale represents a compromise between two conflicting requirements for obtaining accurate gas distribution models: using as many measurements as possible and using only very recent measurements. We have studied several recency functions in a time-dependent extension of the Kernel $\mathrm{DM}+\mathrm{V}^{1}$ algorithm (TD Kernel $\mathrm{DM}+\mathrm{V}^{2}$ ). Based on real-world experiments and simulations of gas dispersal (presented in this paper) we demonstrate that TD Kernel DM+V improves the obtained gas distribution models in dynamic situations. This represents an important step towards statistical modelling of evolving gas distributions.

\section{Methods and Results}

To build a time-scale dependent model, we apply an extension of the Kernel DM+V algorithm, which we call TD Kernel DM+V. It combines the spatial extrapolation of the basic Kernel DM+V algorithm with temporal extrapolation weighted by a time-dependent term defined with a time-scale factor. The timescale is learned together with the other meta-parameters by optimizing the NLPD (Negative Log Predictive Density) value of the predictive model $^{3}$. To evaluate different time-scale dependent modelling approaches, we have developed a gas dispersal simulation engine ${ }^{4}$ that integrates OpenFOAM fluid flow simulation and a filament-based gas propagation model $^{5}$. Simulated data provide ground truth information to evaluate gas dispersion models.

Two simulation experiments were performed in a wind tunnel $\left(16 \times 4 \mathrm{~m}^{2}\right)$ to simulate the gas dispersion under effect of predominantly laminar and turbulent flow, illustrated in Fig 1(a) and Fig 1(b) respectively. Turbulence is particularly created in one experiment by placing an obstacle in the tunnel. Measurements in both experiments have been collected at random locations for 16s from the beginning of gas emission (with the frequency of $1 \mathrm{~s}^{-1}$ ). A predictive model is learned by cross-validation over the training set, optimizing the NLPD value. The model is then used to estimate the gas distribution for the 20th second. The corresponding predictive mean (top) and predictive variance (bottom) using Kernel DM+V (left) and TD Kernel DM+V (right) are illustrated for the first and second experiment in Fig 2 and Fig 3 respectively. The NLPD comparison presented in Tab. 1 shows a substantial improvement with the proposed time-scale dependent approach.

\footnotetext{
${ }^{1}$ A. J. Lilienthal, M. Reggente, M. Trincavelli, J. L. Blanco, and J. Gonzalez, “A Statistical Approach to Gas Distribution Modelling with Mobile Robots - The Kernel DM+V Algorithm”. Proc. IROS, 2009, pp. $570-576$.

${ }^{2}$ S. Asadi, M. Reggente, C. Stachniss, C. Plagemann, and A. J. Lilienthal. "Statistical Gas Distribution Modelling Using Kernel Methods". Chapter in Intelligent Systems for Machine Olfaction (ISMO), 2010.

${ }^{3}$ A. J. Lilienthal, S. Asadi and M. Reggente, "Estimating Predictive Variance for Statistical Gas Distribution Modelling", AIP Conf. Proc. Vol. 1137: Olfaction and Electronic Nose-Proc. of the Int. Symposium on Olfaction and Electronic Nose (ISOEN), 65-68 (2009).

${ }^{4}$ S. Pashami, S. Asadi, and A. J. Lilienthal, "Integration of OpenFOAM Flow Simulation and Filament-Based Gas Propagation Models for Gas Dispersion Simulation”, In: Proc. of the Open Source CFD Int. Conf., 2010.

${ }^{5}$ J. A. Farrell, J. W. Li, and R. T. Cardé, "Filament-Based Atmospheric Dispersion Model to Achieve Short TimeScale Structure of Odor Plumes", Environmental Fluid Mechanics 2, 143-169 (2002).
} 

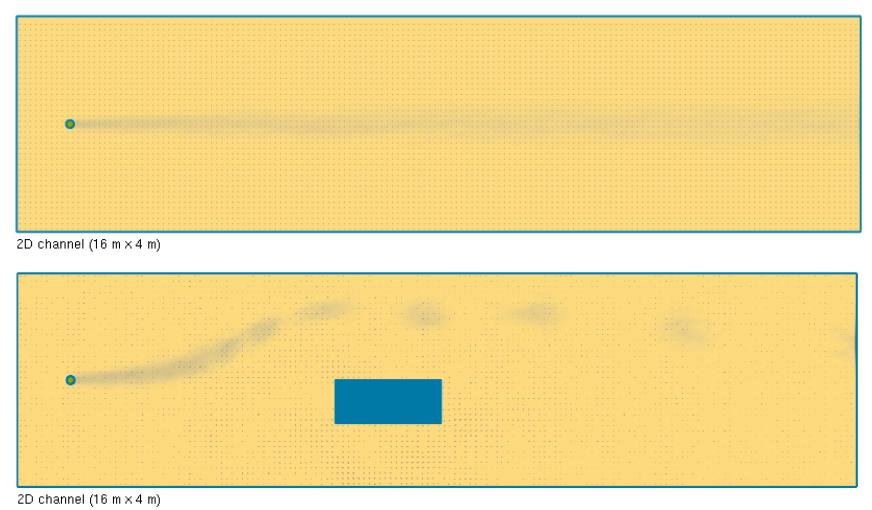

Figure 1: Gas dispersal simulation of the two experiments performed in the wind tunnel $\left(16 \times 4 \mathrm{~m}^{2}\right)$ with the inlet on the left (approx. $1 \mathrm{~m} / \mathrm{s}$ ): (a) gas dispersion in predominantly laminar flow with no obstacle and (b) gas dispersion in turbulent flow created by placing an obstacle in the tunnel (the blue rectangle). An ethanol gas source is placed at $(1 \mathrm{~m}, 2 \mathrm{~m})$ from the bottom left corner indicated by a filled circle.

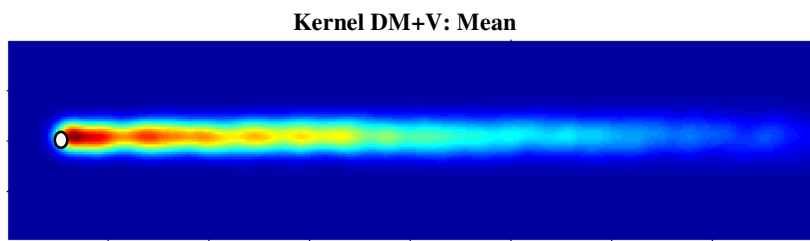

Kernel DM+V: Variance

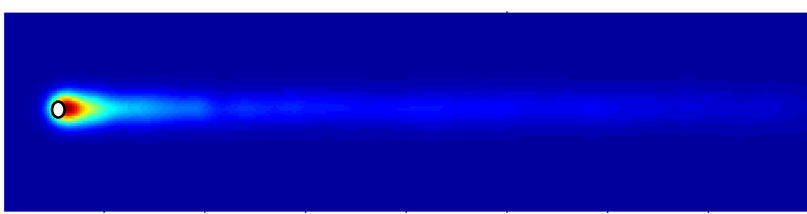

TD Kernel DM+V: Mean

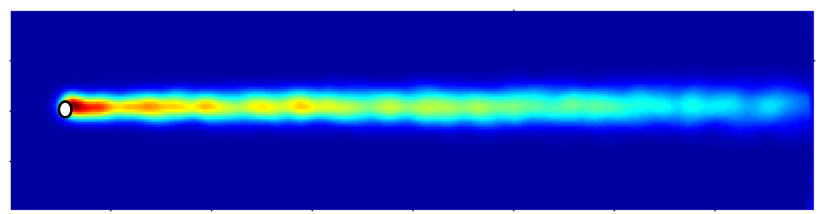

TD Kernel DM+V: Variance

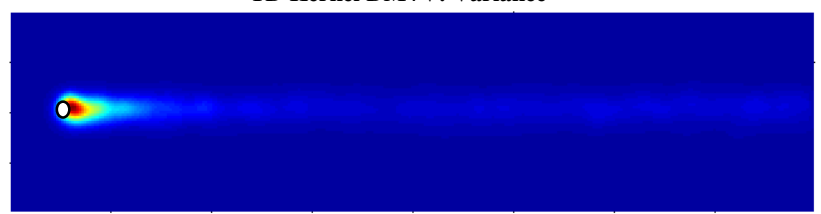

Figure 2: Predicative mean (top) and variance (bottom) maps created for the first experiment shown in Fig 1(a): Kernel DM+V (left) and TD Kernel DM+V (right). The gas source location is indicated by white circle.

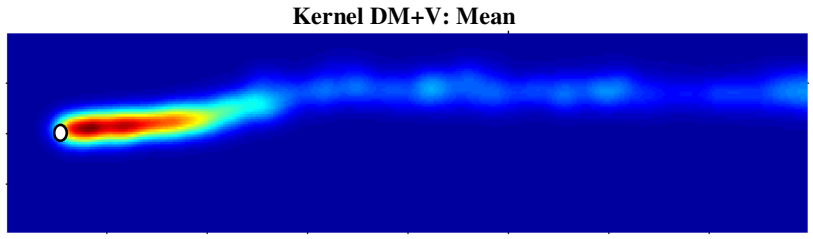

Kernel DM+V: Variance

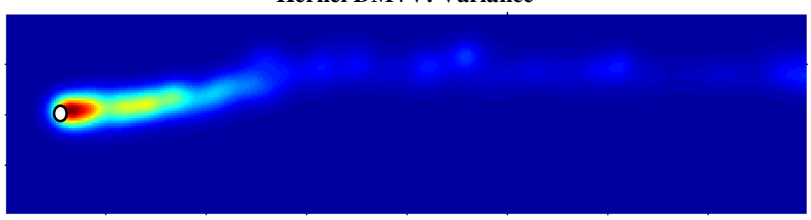

TD Kernel DM+V: Mean

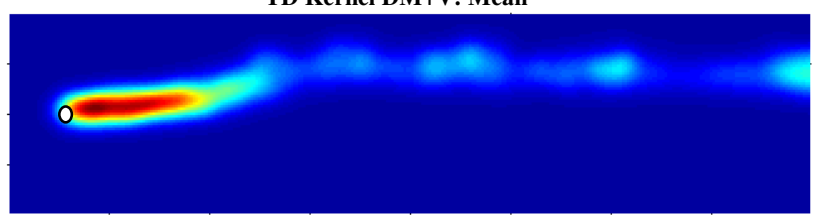

TD Kernel DM+V: Variance

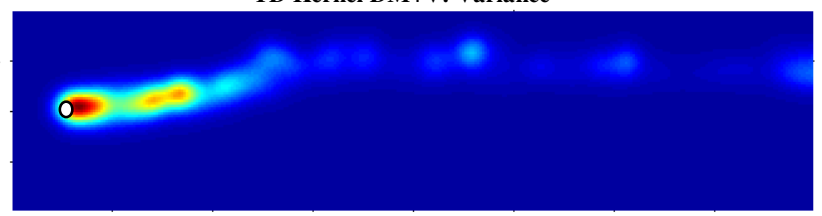

Figure 3: Predictive mean (top) and variance (bottom) maps created for the second experiment shown in Fig 1(b): Kernel DM+V (left) and TD Kernel DM+V (right). The gas source location is indicated by white circle.

Table 1: NLPD comparisons of models built with Kernel DM+V and TD Kernel DM+V for the two experiments: shown in Fig 1 (a) and Fig 1 (b). More negative NLPD values correspond to better gas distribution predictions.

\begin{tabular}{|c|c|c|c|c|c|c|c|}
\hline \multirow{2}{*}{ Experiment } & \multicolumn{3}{|c|}{ Kernel DM+V } & \multicolumn{4}{c|}{ TD Kernel DM+V } \\
\cline { 2 - 7 } & NLPD & $\begin{array}{c}\text { kernel size } \\
(\mathbf{m})\end{array}$ & $\begin{array}{c}\text { cell size } \\
(\mathbf{m})\end{array}$ & NLPD & $\begin{array}{c}\text { kernel size } \\
(\mathbf{m})\end{array}$ & $\begin{array}{c}\text { cell size } \\
(\mathbf{m})\end{array}$ & $\begin{array}{c}\text { time scale factor } \\
\left(\mathbf{s}^{-1}\right)\end{array}$ \\
\hline No obstacle & -6.43 & 0.200 & 0.050 & -14.43 & 0.160 & 0.074 & 0.218 \\
\hline With obstacle & -5.80 & 0.200 & 0.050 & -11.97 & 0.209 & 0.050 & 0.235 \\
\hline
\end{tabular}

